DOI: $\mathrm{xxx} / \mathrm{xxxx}$

\title{
Computational modeling of the fluid flow and the flexible intimal flap in type B aortic dissection via a monolithic Arbitrary Lagrangian/Eulerian fluid-structure interaction model
}

\author{
Pavel Ryzhakov*1 | Eduardo Soudah ${ }^{1,2} \mid$ Narges Dialami ${ }^{1,2}$
}

\author{
${ }^{1}$ Centre Internacional de Mètodes Numèrics \\ en Enginerya, CIMNE, Barcelona, Spain \\ ${ }^{2}$ Universitat Politècnica de Catalunya, UPC, \\ Barcelona, Spain \\ Correspondence \\ *Email: pryzhakov@cimne.upc.edu \\ Present Address \\ CIMNE, C. Gran Capitan s/n, Edificio C1, \\ Campus Nort, UPC, 08034 Barcelona
}

\begin{abstract}
Summary
In the present work we perform fluid-structure interaction simulation of the fluid flow in type B aortic dissection (AD), accounting for the flexibility of the intimal flap. The interaction of the flow with the intimal flap is modeled using a monolithic Arbitrary Lagrangian/Eulerian (ALE) model. The model relies on choosing velocity as the kinematic variable in both domains (fluid and solid) facilitating the coupling. The fluid flow velocity and pressure evolution at different locations is studied and compared against the experimental evidence and the formerly published numerical simulation results. Several tear configurations are analyzed. Flap deformation and stresses are estimated. Influence of the tear size upon the fluid flow and the flap deformation is discussed.
\end{abstract}

\section{KEYWORDS:}

aortic dissection, phantom, CFD, ALE, monolithic, fluid-structure interaction, biomechanics, open source

\section{1 | INTRODUCTION}

An aortic dissection $(\mathrm{AD})$ results from tearing of the inner layer of the aorta. Blood flow emerging through the tear causes dissection of the inner and middle layers of the aorta. If the blood-filled channel ruptures through the outer aortic wall, $A D$ often becomes fatal. Depending on the location of the dissection, one distinguishes between the ascending AD (type A) and descending $\mathrm{AD}$ (type $\mathrm{B}$ ). Type A ADs typically require surgical interventions, while type $\mathrm{B}$ becomes a chronic disease with a high long-term morbidity and mortality rates due to often recurrence, progressive dilatation of lumen and aortic rupture ${ }^{1}$. The survival rates of $\mathrm{ADs}$ of type $\mathrm{B}$ are as follows: from 50 to $80 \%$ at five years and from 30 to $60 \%$ at 10 years. There are several causes for the progressive dilatation of the $\mathrm{AD}$ such as number, location and size of the intimal tears, elasticity of the intimal flap (IF), the pressure differences between the true(TL) and false lumen(FL) or the high intra-luminal pressure that may provoke the rupture of the tissue due to high mechanical stress caused ${ }^{2}$.

Identifying the possibility of high pressure occurrence and/or the impact of the tears on the dissection flow are essential for preventing the lethal outcomes. However measuring or quantifying these parameters clinically is very challenging as it requires invasive techniques. For this reason, numerical simulations and ex-vivo experiments can play an important role in studying the chronic AD progression in order to prevent lethal outcomes. Considerable amount of work has been dedicated to understanding the haemodynamics in the aortic dissection in ex-vivo models ${ }^{3,4}$, experimental phantoms $s^{5,6,7,8,}$, using numerical simulations of phantom models ${ }^{9}, 100$ of real AD patients $\frac{11,12,13,14}{14}$ and most recently using 4D phase-contrast MRI AD data ${ }^{15,}, 16$. 
Majority of the previous numerical simulations of AD considered fluid dynamics only, excluding the elastic effects in the walls and the intimal flap (e.g. $13,10,9)$. However, solid deformation (particularly in the vicinity of the tears) may have considerable effect upon the flow. Moreover, numerical models based on the assumption of rigid solid are incapable of predicting stress distribution within the flap and identify the regions potentially prone to rupture. Therefore, fluid-structure interaction simulations appear to be necessary to accurately reveal the complex nature of the considered phenomena. Few recent works performed FSI in $\mathrm{AD}^{14,12}$. In ${ }^{14}$ partitioned FSI simulation of acute aortic dissection were performed using ABAQUS software and compared against an experiment made on a fraction of porcine aorta connected to an electronically actuated pulsatile flow pump. The analysis concentrated on flow velocities in true and false lumina. Partitioned FSI simulations using ANSYS of aortic dissection based on a sample patient case were performed in ${ }^{12}$. The study obtained predictions of wall shear stress significantly different from those obtained by the rigid wall model. FSI models applied to-date to AD studies fall into the class of partitioned approaches, which often experience low coupling convergence rates (or no convergence) when the density of the interacting fluid and solid have similar densities (which is the case in the problem at hand), known as the 'added-mass-effect' 17] [18. Monolithic FSI models are free of added-mass effect and provide a feasible alternative to the partitioned approaches precisely for the cases characterized by similar densities of the solid and the fluid. As shown in e.g. ${ }^{19}$ or ${ }^{20}$ for Lagrangian class of FSI models, monolithic approaches can be computationally efficient provided that the kinematic variables describing the fluid and the solid are the same.

Current work presents a modeling methodology capable of predicting the coupled fluid-solid behavior following a monolithic approach. The computational model is based on an Arbitrary Lagrangian/Eulerian framework. The formulation utilizes velocity as the unique kinematic variable in both the fluid and the solid, facilitating the monolithic coupling. In the simulations performed particular emphasis is given to the study of the influence of the tear size upon the mechanical response. Remark, that one of the commonly used surgical approaches to AD treatment consists in introducing an additional tear in the intimal flap. However, the exact correlation between the tear size, number of tears and location with the heamodynamics inside the aorta as well as response of the tissue is yet to be fully understood. Therefore, better understanding the impact of the tear size/location can be crucial for the success of the surgical intervention.

The paper is organized as follows. Section 2 presents the governing equations of the fluid-structure interaction model used for the simulation of an AD. Next, in Section 3 geometry, material data and boundary conditions corresponding to the considered $\mathrm{AD}$ case are presented. In Section 4 the most significant results of the numerical simulation are summarized and the comparison with the reference data is made. Section 5 is devoted to summary and conclusions.

\section{2 | COMPUTATIONAL MODEL}

\section{1 | Monolithic ALE fluid-structure interaction approach}

In the present work a monolithic computational model "tailored" specifically for meeting the peculiarities of the problem at hand is implemented. It follows the idea originally proposed in ${ }^{21}$ for fluid flows in biological systems (similar partitioned ALE FSI approach may be found in 22 ). The approach is particularly suitable for modeling systems involving flows in flexible pipes (characterized by solid deformations predominantly in the direction orthogonal to that of the flow). The model relies on solving the fluid-structure interaction problem (blood-flap interactions) within a framework of Arbitrary Lagrangian/Eulerian finite element method (ALE FEM). It belongs to the monolithic type in the sense that the fluid and the solid are marched in time together on a single mesh and no coupling iterations are needed. In order to avoid the necessity of introducing the interface governing equations (ensuring continuity of the kinematic variables and stresses across the fluid-solid boundary), same kinematic variable is used to formulate the governing equations of the fluid and the solid domain following the ideas proposed in the context of Lagrangian FSI models in ${ }^{19}$ and $\mathrm{d}^{23}$. This distinguishes the present approach from 21 , where difference in the kinematic variables between the solid and the fluid domain obliged introducing additional interface equations.

The overall ALE FSI setting is shown in Fig. 1 For explaining the concept, let us consider a model problem: a fluid flow in a flexible pipe. Fig. 1 (a) shows the discretized domain consisting of a fluid between a rigid lower and a flexible upper wall. A constant flux is prescribed on the left (inlet), the flexible upper wall is fixed at its left edge. The fluid flow causes deformation in the solid. The deformed configuration is shown in Fig. 1 (b) One can see that the fluid elements in contact with the solid deform together with the solid, while the rest of the fluid elements remain undeformed. This is facilitated by using the Arbitrary Lagrangian/Eulerian (ALE) framework, which encompasses Lagrangian and Eulerian ones as two limiting cases. Grey part of the domain $\left(\Omega_{L}\right)$ corresponds to the solid, that is described in the Lagrangian fashion. This is obtained in the general ALE framework 


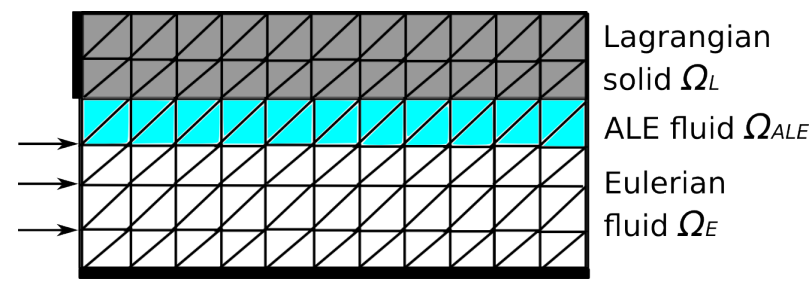

(a) Undeformed configuration

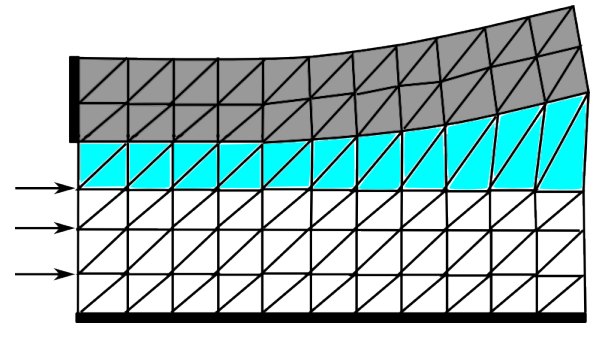

(b) Deformed configuration

FIGURE 1 Different sub-domains of the monolithic ALE fluid-structure interaction model

by setting mesh velocity equal to material velocity. White part of the domain $\left(\Omega_{E}\right)$ corresponds to the fluid, that is described within the fixed grid (Eulerian framework obtained in ALE by setting mesh velocity to zero) except for the layer of elements adjacent to the solid $\left(\Omega_{A L E}\right)$. Interfacial fluid elements are treated as follows: the nodes shared with the solid move according to their convective velocity ("Lagrangian nodes"), while the rest of the nodes are maintained fixed ("Eulerian nodes"). Thus, the overall setting is the Arbitrary Lagrangian/Eulerian one, where different sub-domains have different relationship between the mesh velocity and the material velocity.

In the following the governing equations will be presented. Details of the monolithic ALE FSI formulation used here (including the discretized equations) can be consulted in 24 .

\section{2 | Governing equations}

In both the fluid and the solid the linear momentum conservation equation holds. In the Arbitrary Lagrangian/Eulerian framework this equation can be written as

$$
\rho \frac{\partial \mathbf{v}}{\partial t}+\rho(\mathbf{c} \cdot \nabla) \mathbf{v}-\nabla \cdot \sigma=\mathbf{f}_{e x t}
$$

where $\mathbf{v}$ is the material velocity, $\mathbf{c}$ is the convective velocity with respect to the ALE mesh moving with velocity $\mathbf{v}_{m}$ (such that $\mathbf{c}=\mathbf{v}-\mathbf{v}_{m}$ ), $\sigma$ is the Cauchy stress, and $\mathbf{f}_{\text {ext }}$ is an external force (typically, gravity). Density of the continuum is denoted as $\rho$.

\section{Fluid model}

The fluid flow in the application of interest is either blood (real AD) or phantom fluid (water in the in vitro AD experiment we are studying). The fluid will be modeled as a viscous incompressible Newtonian fluid. Thus, additionally to the momentum conservation (Eq. 1) the incompressibility condition must be satisfied:

$$
\nabla \cdot \mathbf{v}=0
$$

The constitutive equation for Newtonian fluid reads:

$$
\sigma=-\rho k \nabla \cdot\left(\nabla \mathbf{v}+\nabla \mathbf{v}^{T}\right)+p I
$$

where $k$ is the kinematic viscosity, $p$ is the fluid pressure and $I$ is the identity matrix.

\section{Solid model}

The intimal flap material (silicon in the in vitro experiment) exhibits a nearly linear elastic behavior. In order to model this behavior and at the same time match the kinematic variable of the fluid (so as to ensure that the solid and the fluid domains have the same degrees of freedom at shared nodes and thus automatically move together), we shall use the hypo-elastic solid model. This model can be naturally used in conjunction with the momentum equation (Eq.11) written in terms of velocity. We note that in the solid elements the convective velocity equals the material velocity $(\mathbf{c}=\mathbf{v})$ making the convective term of the momentum equation vanish. 
An isotropic hypoelastic solid (see e.g. ${ }^{25}$ ) is a material defined by a linear relation between the stress rate and the deformation rate:

$$
\stackrel{\circ}{\tau}=2 \mu D+\lambda(\operatorname{tr} D) I
$$

where $D=\frac{\nabla \mathbf{v}+\nabla \mathbf{v}^{T}}{2}$ is the strain rate tensor and $\mu$ and $\lambda$ are the Lame constants that can be expressed as functions of Young modulus $E$ and Poisson's ratio $v$ of the material as:

$$
\begin{array}{r}
\mu=\frac{E v}{(1+v)(1-2 v)} \\
\lambda=\frac{E}{1+v}
\end{array}
$$

$\stackrel{\circ}{\tau}$ is known as Truesdell rate of Kirchhoff stress tensor $\tau$, which is related to the rate of second Piola-Kirchhoff stress $S$ as:

$$
\stackrel{\circ}{\tau}=F \dot{S} F^{T}
$$

where $F$ is the deformation gradient. Cauchy stress is related to Piola-Kirchhoff stress as

$$
\sigma=J^{-1} F S F^{T}
$$

where $J$ is the Jacobian (determinant of the deformation gradient).

Adopting the updated Lagrangian framework, considering that the current unknown configuration is the reference, the above equation leads to the following expression for the time derivative of the Cauchy stress tensor (note that the deformation gradient equals unity in this case) ${ }^{26}$ :

$$
\dot{\sigma}=\kappa \epsilon_{v} I+2 \mu D
$$

where $\epsilon_{v}=\operatorname{tr}(D)$ is the volumetric strain rate and $\kappa=\lambda+\frac{2}{3} \mu$ is the volumetric viscosity. The Cauchy stress $\sigma$ is obtained from the stress rate by integrating Eq. 10 in time.

\section{3 | MODEL DATA}

\section{Geometry and material properties}

As a reference the experimental data of the in vitro study ${ }^{6}$ is used here. In the mentioned experiment, the phantom models of dissected aorta were made of silicon (TL wall) and latex (FL outer wall) and connected to a dynamic flow circuit, mimicking the cardiovascular system. The models exclude the aortic arch and the true lumen has a constant inner diameter. To simulate proximal and distal tears, circular holes of various diameter were created (see Fig. 2. The phantom is fixed at the proximal and distal parts by fixation rings. The dimensions are as follows: true lumen inner diameter D1=14 $\mathrm{mm}$, dissected segment diameter $\mathrm{D} 2=40 \mathrm{~mm}$, false lumen length $\mathrm{L} 1=160 \mathrm{~mm}$, dissection flap thickness $\mathrm{h} 1=2 \mathrm{~mm}$, true lumen lenth $\mathrm{L} 2=390 \mathrm{~mm}$. Centers of the circular holes representing the tears were located at a distance of $175.5 \mathrm{~mm}$ (proximal) and $320.5 \mathrm{~mm}$ (distal) from the inlet. With respect to tear diameters, three scenarios were considered. In the first one (this will be referred here-thereafter as "Case 10-10") both tears had a diameter of $10 \mathrm{~mm}$, in the second one ("Case 10-4"), proximal tear had a diameter of $10 \mathrm{~mm}$, while the diameter of the distal one was $4 \mathrm{~mm}$. An additional case with only a proximal tear of $10 \mathrm{~mm}$ diameter ("Case 10-0") was also analyzed with the objective to understand the effects of the secondary tear upon the dissection flow. The geometrical models reproducing these three configurations are shown in Fig 3 and summarized in Table 1 .

\section{Inlet, outlet and wall conditions}

Boundary conditions used in the present work were prescribed according to the data of the in vitro experiments.

At a point $\mathbf{x}$ of the fluid inlet a parabolic time-dependent velocity profile is prescribed:

$$
\mathbf{v}(\mathbf{x}, t)=\mathbf{n} v_{p r}(t)\left(1-\left(\frac{r(\mathbf{x})}{R}\right)^{2}\right)
$$

where $\mathbf{n}$ is the unit normal vector to inlet surface, $r$ is the distance from the center of the inlet, $R$ is the inlet radius and $v_{p r}$ is the velocity magnitude obtained experimentally.

At the outlet time-dependent Neumann condition was prescribed: 


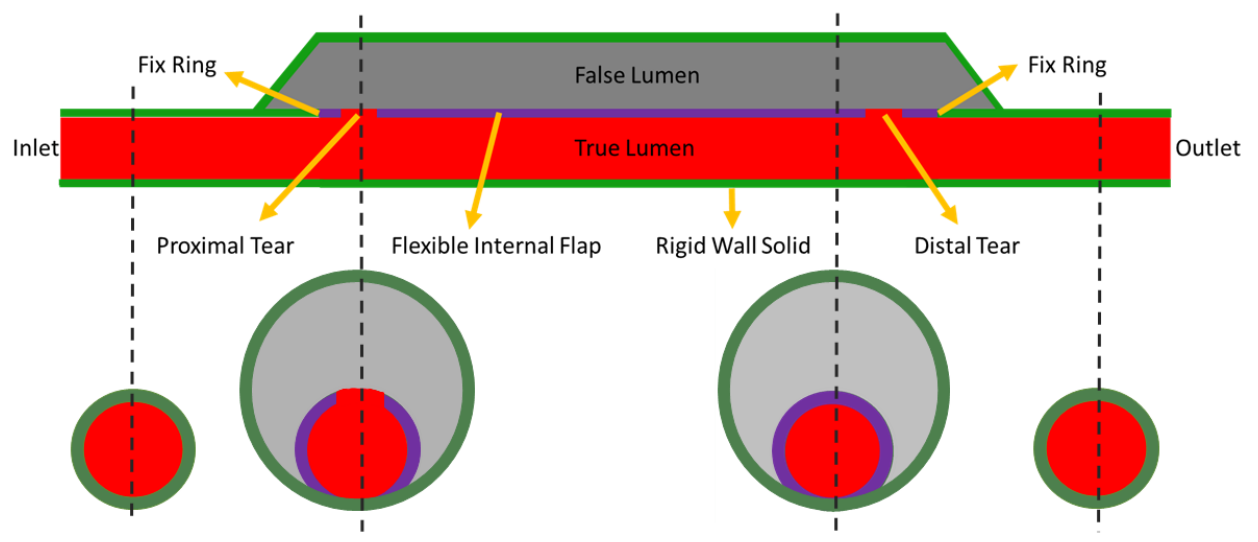

FIGURE 2 Synthetic model of a type B aortic dissection. Cross sectional areas: inlet, proximal tear, distal part and outlet

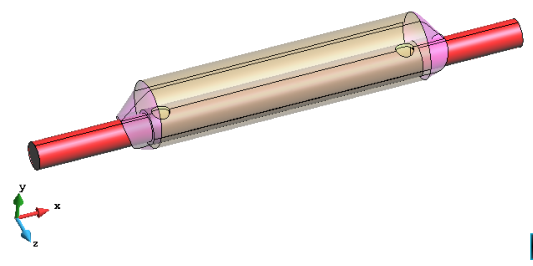

(a) Case 10-10

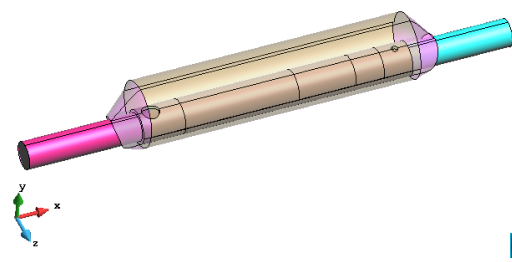

(b) Case 10-4

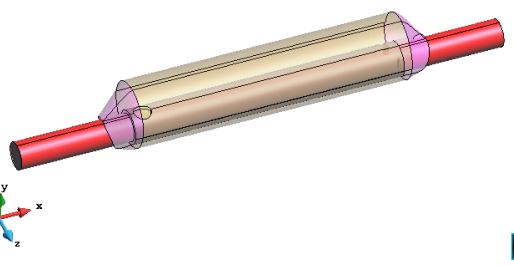

(c) Case 10-0

FIGURE 3 Analyzed configurations.

$$
\boldsymbol{\sigma} \cdot \mathbf{n}=p_{p r}(t)
$$

Time-dependent prescribed values $\left.\mathbf{v}_{p r}(t)\right)$ and $p_{p r}(t)$ are specified in Fig. 4 In our work we impose the pressure profile measured at the outlet by ${ }^{6}$. To make consistent comparisons and with the objective to analyze the influence of the distal tear, in our study same inlet waveform and outlet pressure were considered in all cases.

The pressure input (Fig. 4 b)) represents a period corresponding to normal haemodynamic conditions in the human aorta. The duration of the period is about $0.88 \mathrm{~s}$, with the peak pressures reached at $0.25 \mathrm{~s}$ approximately and a biphasic diastolic period. The perfusion liquid used in the experiment was distilled water, with density of $1000 \mathrm{~kg} / \mathrm{m}^{3}$ and kinematic viscosity of $0.86 \times 10^{-6} \mathrm{~m}^{2} / \mathrm{s}$.

AD walls are considered rigid (green), with the exception of the intimal flap (purple) (see Fig. 2). The mechanical properties of the flexible intimal flap are:

- Young's modulus $E=2 \mathrm{KPa}$.

- Poisson's ration: $v=0.4$

- Density $\rho=2000 \mathrm{~kg} / \mathrm{m}^{3}$

The bottom part of the AD and the proximal/distal joint areas (ring) of the intimal flap with the aorta are also fixed, see Fig. 2 Homogeneous velocity boundary condition $(\mathbf{v}=0)$ is prescribed at all the outer walls, except for the one separating the true lumen and the false lumen. 


\begin{tabular}{lcc}
\hline Case & Proximal tear $(\mathbf{m m})$ & Distal tear $(\mathbf{m m})$ \\
\hline Case $10-10$ & 10 & 10 \\
Case $10-4$ & 10 & 4 \\
Case $10-0$ & 10 & 0 \\
\hline
\end{tabular}

TABLE 1 Cases studied.

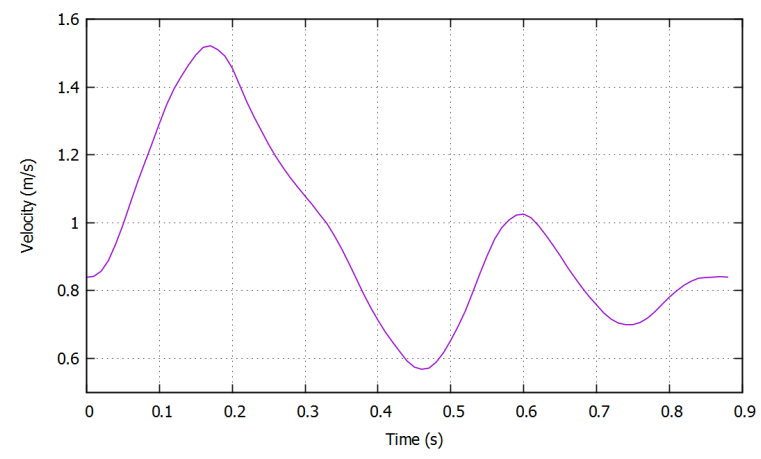

(a) Velocity at the inlet

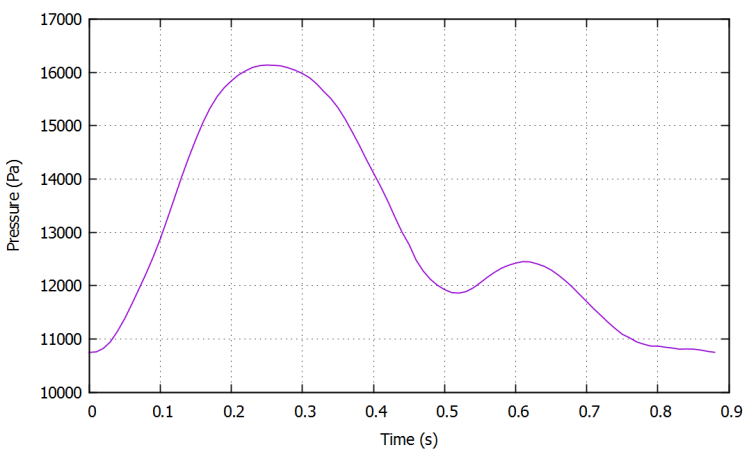

(b) Pressure at the outlet

FIGURE 4 Time-dependent inlet and outlet boundary conditions.

\section{4 | RESULTS OF THE NUMERICAL SIMULATION}

In the present section the AD FSI simulation results obtained using the present numerical model are discussed. The ALE FSI model was implemented by the authors in the ULFApplication branch of Kratos Multiphysics, an Open Source C++ object oriented Finite Element framework ${ }^{27}$. The generalized Newton-Raphson procedure was used to solve the governing system of equations. At every non-linear iteration, the monolithic linear system (solid+fluid) was solved using the stabilized Bi-conjugate gradient method (BiCGSTAB). The convergence criteria used were as follows:

- non-linear iterations tolerances

$$
\begin{aligned}
& \text { Velocity. Relative: } \epsilon_{v}=\frac{\|\delta \mathbf{v}\|}{\left\|\mathbf{v}_{n+1}\right\|}<10^{-6} \text {. Absolute: } 10^{-8} \\
& \text { Pressure. Relative: } \epsilon_{p}=\frac{\|\delta p\|}{\left\|p_{n+1}\right\|}<10^{-4} \text {. Absolute: } 10^{-6}
\end{aligned}
$$

- linear solver tolerances

BiCGSTAB: $10^{-8}$

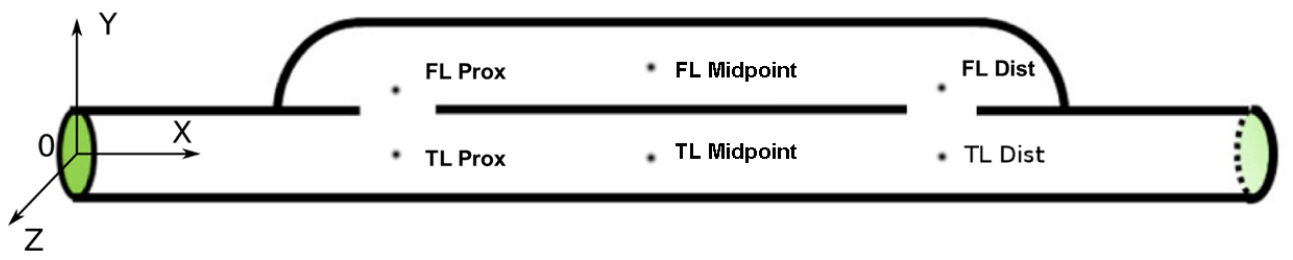

FIGURE 5 Location of the analysis points for the fluid pressure ("numerical sensors"). 


\begin{tabular}{lccc}
\hline Point & $\mathbf{X}$ & $\mathbf{Y}$ & $\mathbf{Z}$ \\
\hline TL Proximal & 0.1755 & 0 & 0 \\
FL Proximal & 0.1755 & 0.017 & 0 \\
TL Distal & 0.3205 & 0.017 & 0 \\
FL Distal & 0.3205 & 0 & 0 \\
TL Midpoint & 0.248 & 0.0 & 0 \\
FL Midpoint & 0.248 & 0.017 & 0 \\
\hline
\end{tabular}

TABLE 2 Location of the analysis points for the fluid pressure ("numerical sensors").

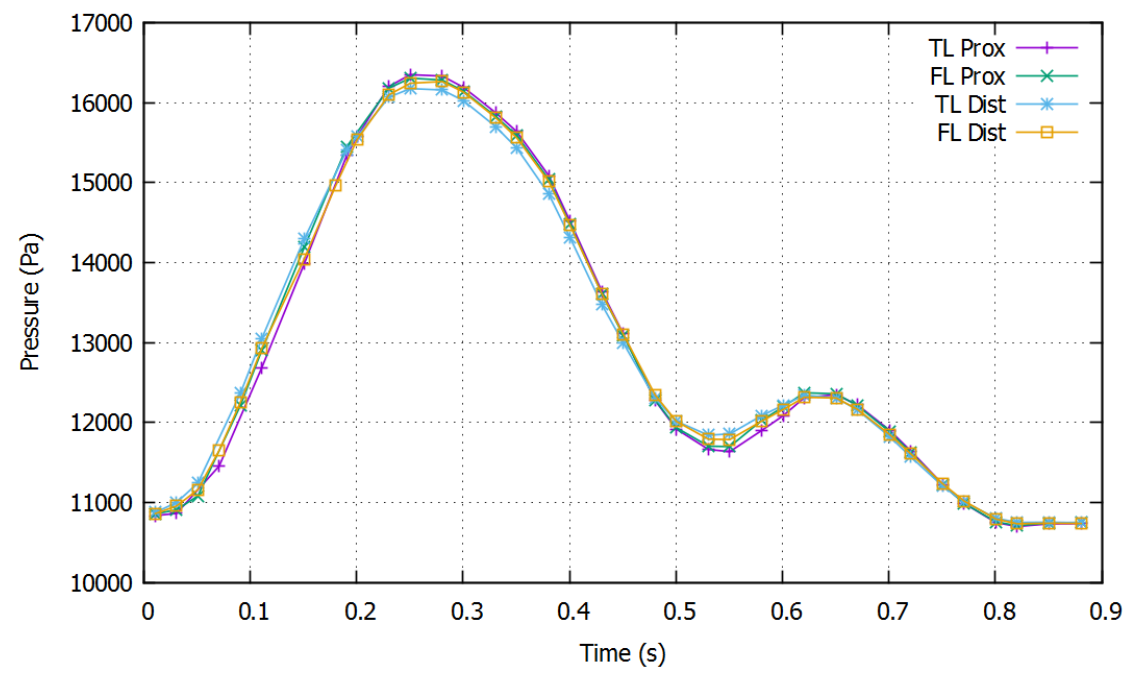

FIGURE 6 Case 10-10. Pressure evolution at the "numerical sensors".

The locations of pressure measurements are indicated on Fig. 5 Additionally, maximum midpoint pressure was recorded in TL and FL (at a mid-distance between the corresponding proximal and distal points). The coordinates of the points are summarized in Table 2 The model is aligned with the $\mathrm{X}$-axis, zero of the coordinate system is located at the center of the inlet.

\section{1 | Case 10-10}

The first configuration analyzed (Case 10-10) involves two tears of equal diameter (10 mm). First, fluid flow is studied and afterwards emphasis is given to the intimal flap deformation.

\section{Fluid flow}

Fig. 6 shows pressure evolution measured at 4 "numerical sensors". One can see that the peak pressure of about $16.2 \mathrm{KPa}$ is reached at time $\mathrm{t}=0.25$. Local minimum (about $12.7 \mathrm{KPa}$ ) is reached at $\mathrm{t}=0.53 \mathrm{~s}$. Both peaks coincide with the ones of the prescribed outlet pressure. Very minor difference is observed among the values recorded at different sensors.

This behavior (nearly the same pressure evolution at all the locations) was also observed in 6 . Fig. 7 shows comparison of the pressure results obtained in the present work with those of the in vitro experiment ${ }^{[6}$ as well the CFD simulation ${ }^{9}$ (the latter performed assuming rigid flap). One can see that the present results nearly coincide with the in vitro data. The peak value during the systole exactly coincides at the true lumen distal sensor, while at the true lumen proximal sensor the peak value obtained in the present work is slightly higher than that of ${ }^{6}$. Similar trend is observed in ${ }^{9}$ and ${ }^{10}$. Negative pressure gradient in X-direction observed in the numerical simulations may be attributed to the pressure boundary conditions which is prescribed at the outlet 


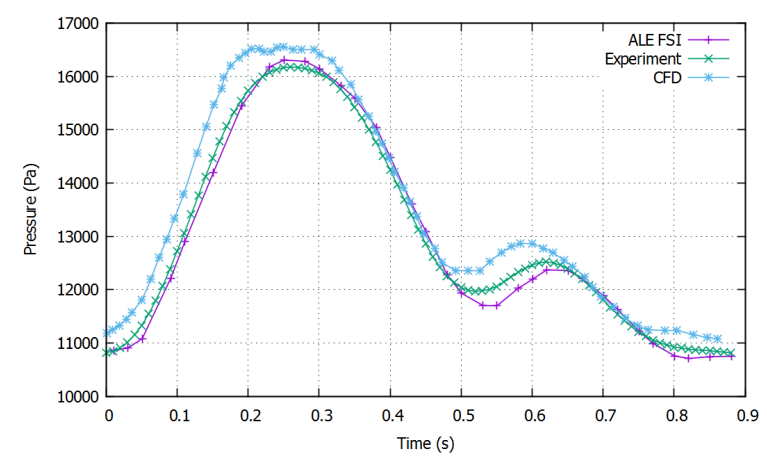

(a) FL proximal pressure

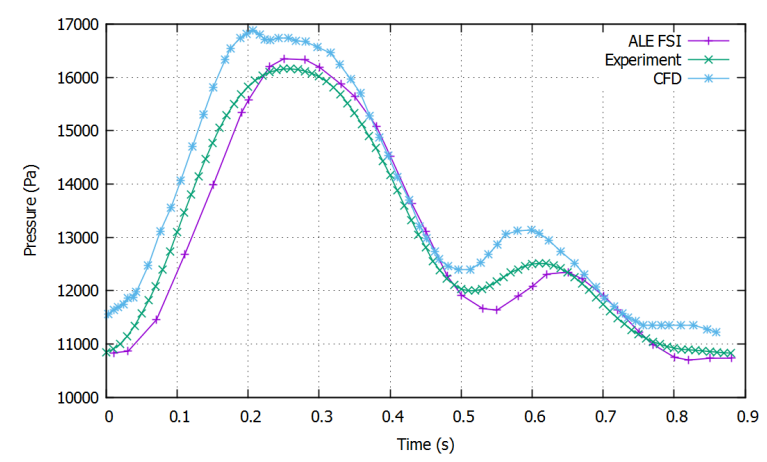

(c) TL proximal pressure

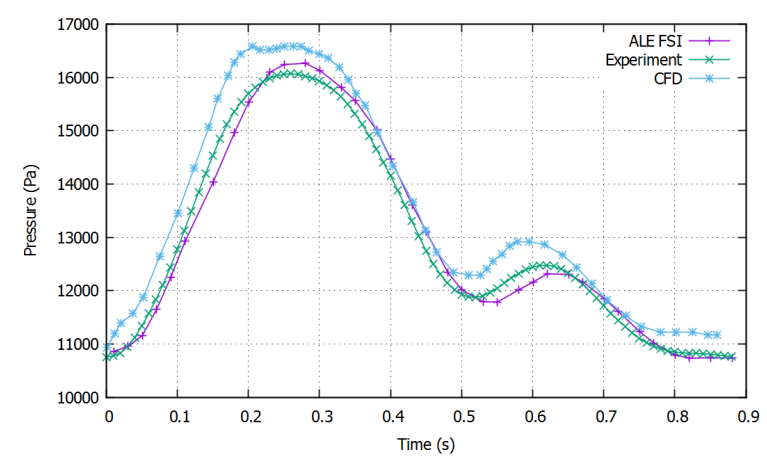

(b) FL distal pressure

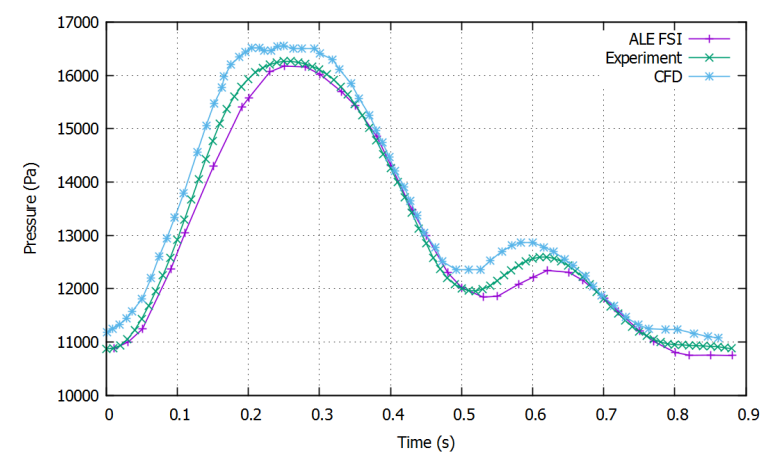

(d) TL distal pressure

FIGURE 7 Case 10-10. Pressure evolution at various locations. Comparison with the results of ${ }^{[6}$ and ${ }^{9}$

(i.e. in the vicinity of the true lumen distal tear). Equal pressure evolution at analyzed locations demonstrates that the large tears ensure that no large pressure gradients between FL and TL arise.

Fig. 8 shows fluid velocity distribution at two time instances: 0.1 and $0.4 \mathrm{~s}$. At these two time instances the highest velocities are observed at the proximal and distal tears, respectively. One can see that the velocity peaks at the tears do not exactly coincide in time with the peaks in the inlet velocity (which correspond to 0.16 (peak systole) and $0.46 \mathrm{~s} \mathrm{(peak} \mathrm{diastole)} \mathrm{approximately).}$

The velocity field is shown along the vertical cut made at $\mathrm{Z}=0$ (XY-plane). One can see velocity fluxes through the tears. At $\mathrm{t}=0.1 \mathrm{~s}$ a flux (maximum velocity of the jet is about $1.35 \mathrm{~m} / \mathrm{s}$ ) enters TL via the proximal tear. At the same moment the fluid is suctioned into the TL via the distal tear (negative vertical velocity of about $1.2 \mathrm{~m} / \mathrm{s}$ at the peak of the jet). It is important to note that the jet is displaced from the center in the downstream direction, i.e. towards the right wall of the tear. At $t=0.4 \mathrm{~s}$, fluid enters the false lumen via the distal tear with a velocity of $1.2 \mathrm{~m} / \mathrm{s}$ and exits the false lumen via the proximal tear at $0.5 \mathrm{~m} / \mathrm{s}$ approximately. The jet through the proximal tear is wide, occupying nearly the entire tear, while the distal tear jet is narrow and shifted towards the right wall of the tear.

Horizontal velocity in the false lumen is barely affected by the flow through the tears, reaching maximum value at the center of the channel, the value being close that that of the inlet velocity. Nearly no vorticity is observed.

The velocity evolution has been recorded in the vicinity of the proximal and the distal tear. We selected the location so as to capture the observed jets $((\mathrm{x}, \mathrm{y}, \mathrm{z})=(0.1806,0.01,0.0)$ for the proximal tear and $(\mathrm{x}, \mathrm{y}, \mathrm{z})=(0.326,0.0067,0.0)$ for the distal one $)$. Comparison of the obtained velocity through the proximal tear with the result of ${ }^{6}$ is shown in Fig. 9 (a) The results of the in vitro experiment shown here were obtained by averaging the highly oscillatory graph provided in ${ }^{6}$. One can see a very similar trend and coincident location of maxima and minima. However, the positive peak values obtained in the present work are larger than the ones measured in ${ }^{6}$. On the other hand, negative values are nearly the same.

This can be explained by the fact that the velocity in the experimental study were measured approximately at the center of the tears, while the jet is actually shifted to the right as can be clearly seen in Fig. 10 A 3D vector plot of the velocity at the 


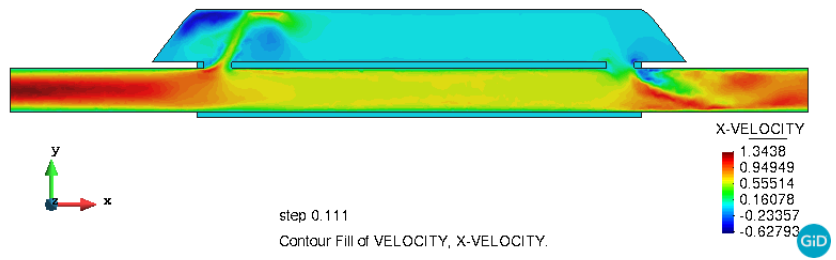

(a) $\mathrm{t}=0.1 \mathrm{~s}$

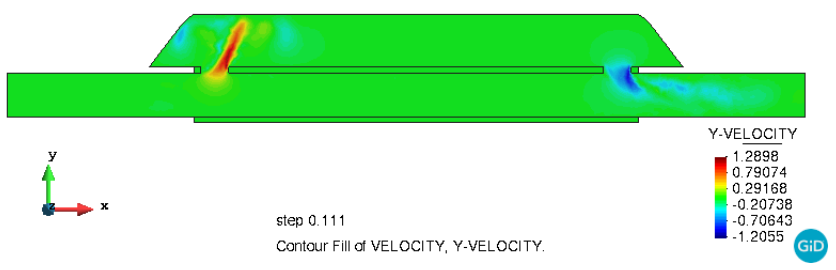

(c) $\mathrm{t}=0.1 \mathrm{~s}$

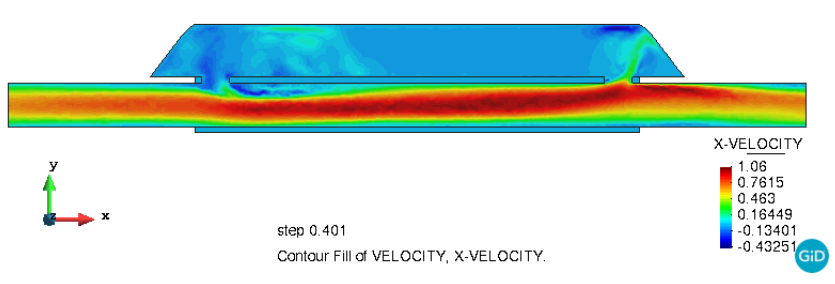

(b) $\mathrm{t}=0.4 \mathrm{~s}$

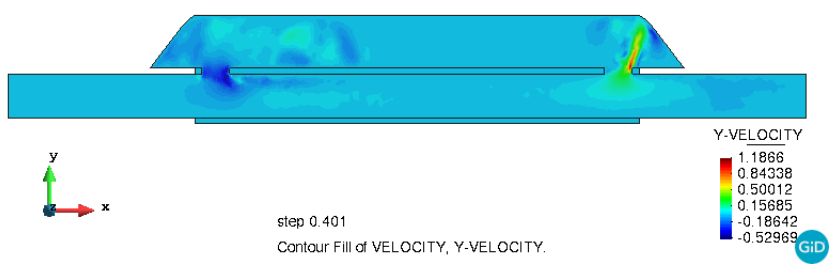

(d) $\mathrm{t}=0.4 \mathrm{~s}$

FIGURE 8 Case 10-10. Velocity fields at different time instances.

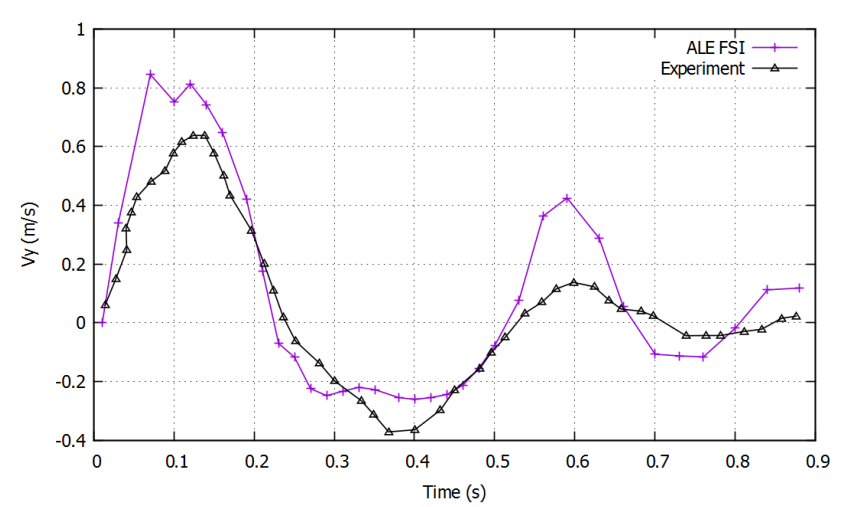

(a) Velocity evolution at proximal tear. Comparison with 6

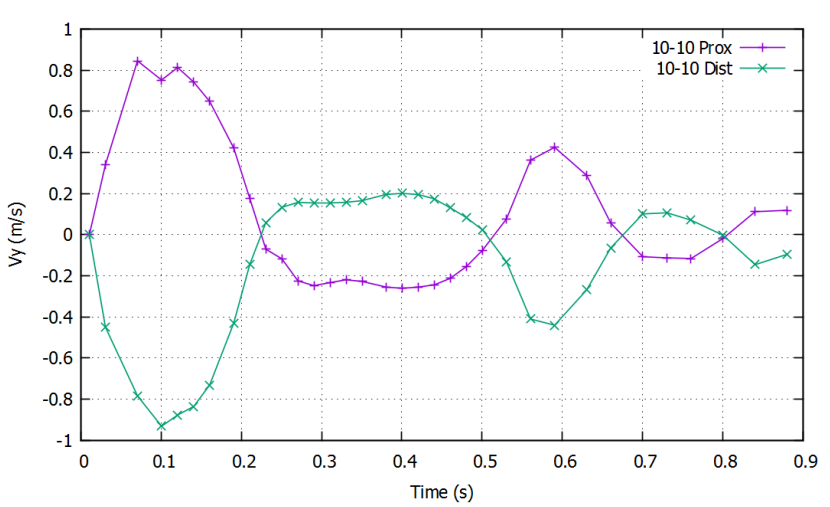

(b) Velocity evolution at proximal and distal tears

FIGURE 9 Case 10-10. Vertical velocity evolution at proximal and distal tears.

proximal tear are shown in Fig. 10 (a) Vertical velocity $\left(V_{y}\right)$ distribution along the longitudinal direction at the proximal tear is shown in Figs. 10 (b) One can see that the velocity at the center of the tear is more than twice lower than the maximum value (located at a distance $d=0.007 \mathrm{~m}$, measured from the left corner of the tear) .

Velocity evolution at the proximal and the distal tears is shown in Fig. 9 . One can see that the fluxes through both tears are very similar in value. Similar results were reported in ${ }^{10}$ and 28 .

The directions of the fluxes through two tears are opposite, that is, when a flux enters the FL via the proximal tear, it exits from there via the distal one and vice versa. Taking into account that the size of the two tears is equivalent and the pressure in the TL and FL are nearly the same, similar fluxes may be expected to be produced at both tears. Similar observations are made in ${ }^{11}$. However, 6 reports that during the systole fluid enters the FL at both the proximal and the distal tear. Such behavior is possible in case of an incompressible fluid flow only if either the FL walls expand (thus accommodating increased volume of fluid) or if the fluid exits the FL simultaneously via the same tears (creating a recirculation at the tears). These phenomena have been observed neither in the experiment nor in the numerical studies. 


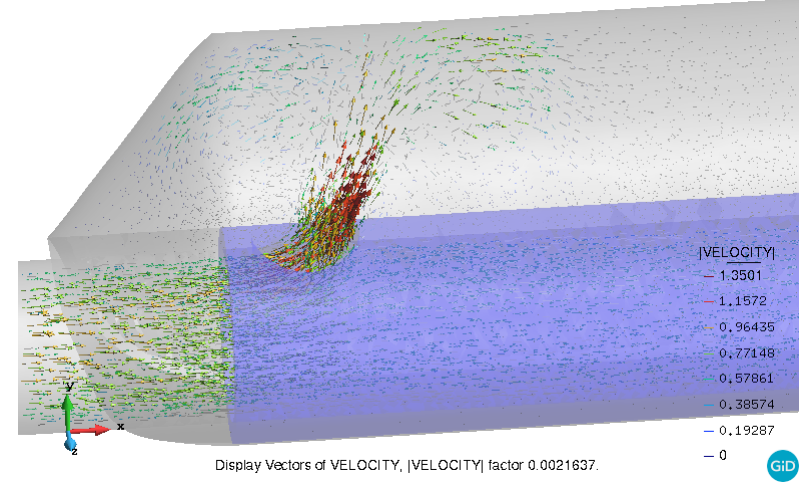

(a) Velocity vectors

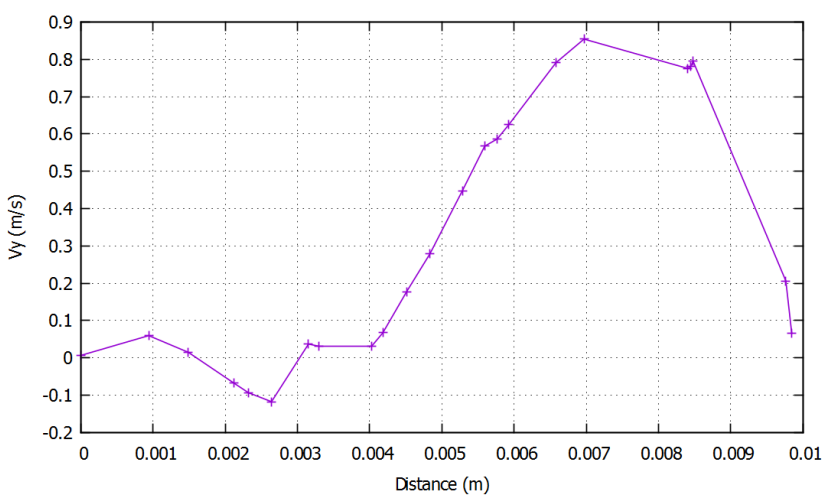

(b) Velocity distribution along the tear (longitudinal direction)

FIGURE 10 Case 10-10. Velocity at proximal tear. $\mathrm{t}=0.1 \mathrm{~s}$

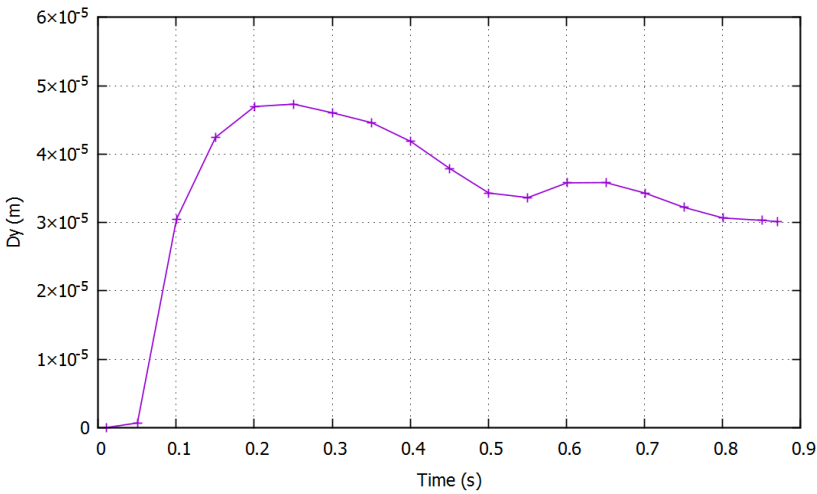

(a) Vertical displacement at Point $\mathrm{M}$

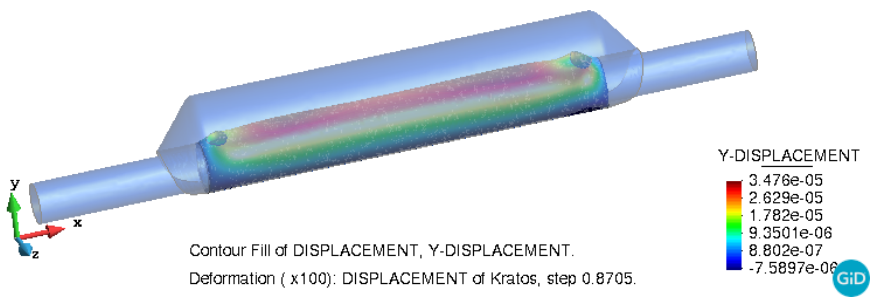

(b) Deformed solid at $\mathrm{t}=0.87 \mathrm{~s}$. Magnif. $\mathrm{x} 100$

FIGURE 11 Case 10-10. Flap deformation.

\section{Flap deformation}

Fig. 11 (a) shows time evolution of the vertical displacement of the the flexible flap measured at the middle of the flap $((\mathrm{x}, \mathrm{y}, \mathrm{z})=(0.25,0.99,0))$. One can see that maximum displacement is reached at $\mathrm{t}=0.25 \mathrm{~s}$, nearly coinciding with the instance of maximum pressure. Maximum displacement equals approximately $0.045 \mathrm{~mm}$, which is relatively small, taking into account that the flap thickness $h_{1}=2 \mathrm{~mm}$ and the outer diameter of the flap tube is approximately $16 \mathrm{~mm}$. Fig. 11 (b) shows the distribution of Y-displacement in the flap at $\mathrm{t}=0.3 \mathrm{~s}$ as well as the deformed geometry at the end of the simulation $(\mathrm{t}=0.88 \mathrm{~s})$. In order to clearly see the deformation in the solid, the displacements were magnified by the factor of 100. One can see expansion of the intimal flap and a symmetric pattern with respect to the $\mathrm{YZ}$ plane placed at $\mathrm{x}=0.25 \mathrm{~m}$. Evolution of the normal stresses in the intimal flap is shown in Section 4.4

In order to assess the impact of accounting for the flap flexibility upon the flow behavior, the simulation using a rigid solid model was performed. It was discovered that nearly no effect upon the fluid flow takes place. Distal FL pressure evolution obtained using the rigid and the FSI model is shown Fig. 12 One can see that solutions are nearly coincident. Taking into account that the maximum displacement observed in the intimal flap is very low, this behavior is expectable. 


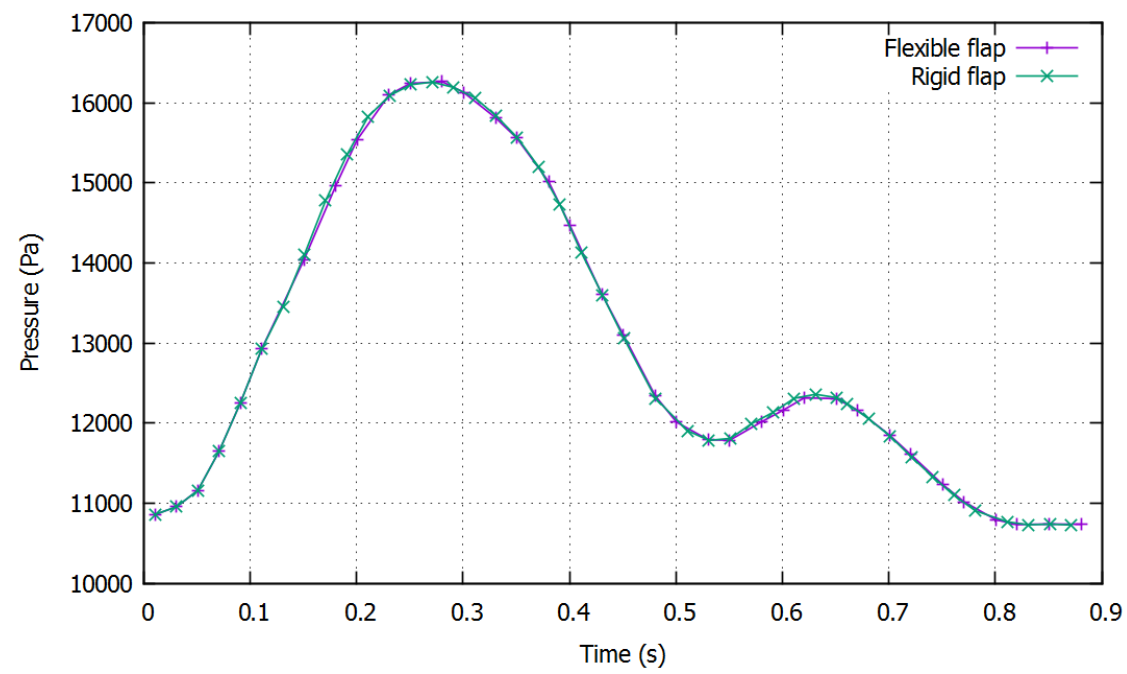

FIGURE 12 Case 10-10. Distal FL pressure evolution. Flexible vs rigid flap.

\section{2 | Case 10-4}

For assessing the effect of the tear size upon the fluid flow and the flap deformation next we consider a configuration with a smaller distal tear. Case 10-4 consists of a proximal tear with a diameter of $10 \mathrm{~mm}$ and a distal tear with a diameter of $4 \mathrm{~mm}$. The inlet and outlet boundary conditions are identical to the ones used for Case 10-10.

\section{Fluid flow}

Fig. 13 shows pressure evolution measured at the numerical sensors. Once again, pressure evolution at all locations closely resembles the prescribed outlet pressure. The peak pressures are slightly higher than the ones observed in the Case 10-10. Additionally, in comparison with the Case 10-10, the difference between the true lumen distal pressure and the pressure at the rest of the locations becomes more evident. Proximal TL and FL as well as distal TL pressures are nearly identical. Smaller distal tear is responsible for the difference between the pressure at the distal TL sensor and the pressure in the rest of the sensors.

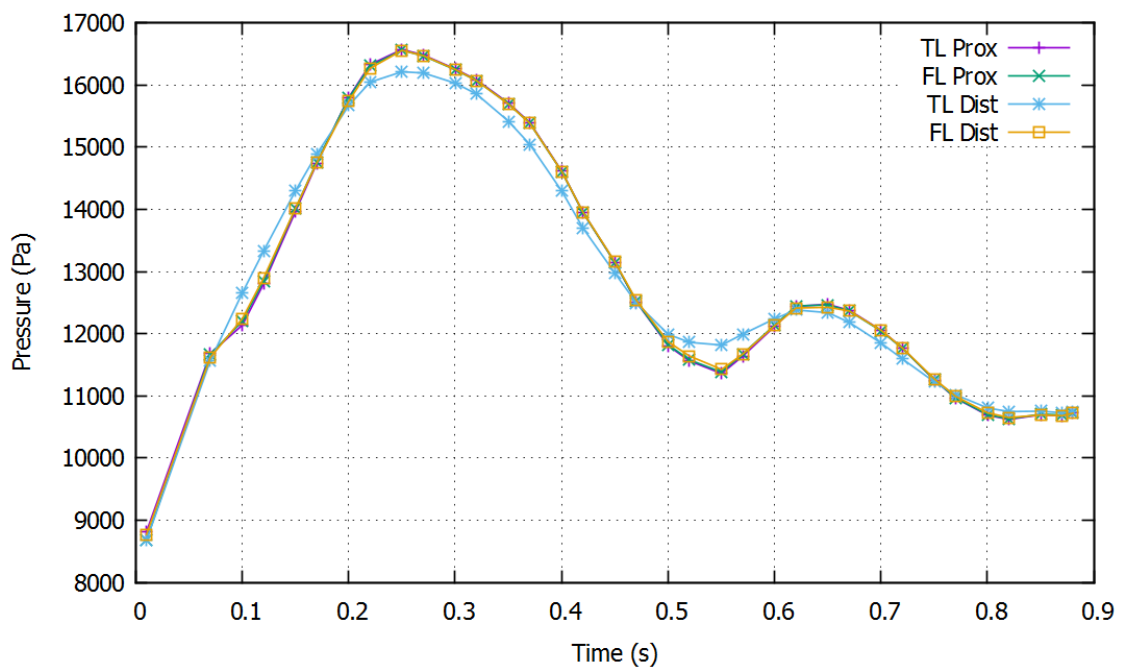

FIGURE 13 Case 10-4. Pressure evolution at the "numerical sensors". 
Fig. 14 shows velocity distribution at two time instances: 0.1 and 0.4 s. Horizontal and vertical velocity fields are shown separately. Similarly to the Case 10-10, at these time instances the highest velocities through the tears are observed. At $\mathrm{t}=0.1 \mathrm{~s}$ a flux with the velocity of $0.8 \mathrm{~m} / \mathrm{s}$ enters FL through the proximal tear. Simultaneously, fluid is enters TL from the FL through the distal tear with the maximum jet velocity of about $1.5 \mathrm{~m} / \mathrm{s}$. Jet at the proximal tear is located at the right corner of the tear (additionally a smaller flow having velocity in opposite direction is formed at the left corner of the tear). Jet at the distal tear is wider and occupies nearly the whole tear. At $t=0.4 \mathrm{~s}$ nearly no flux is observed through the proximal tear, while a jet with the maximum velocity of $1.1 \mathrm{~m} / \mathrm{s}$ enters false lumen through the distal tear. In the formerly analyzed case (10-10) at $\mathrm{t}=0.4 \mathrm{flow}$ from false into true lumen was observed at the proximal tear.

Horizontal velocity in the false lumen is nearly not affected by the flow through the tears, having the maximum value at the center of the channel, the value being close that that of the inlet velocity.

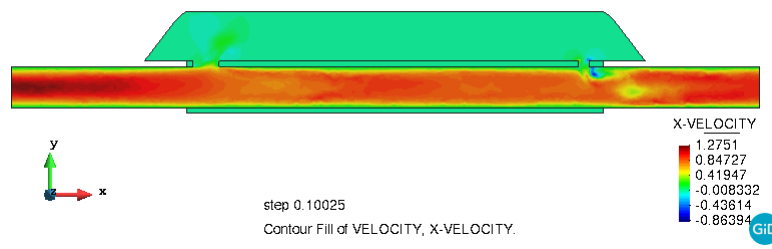

(a) $\mathrm{t}=0.1 \mathrm{~s}$

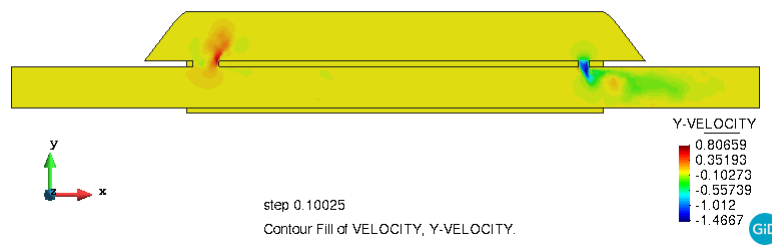

(c) $\mathrm{t}=0.1 \mathrm{~s}$

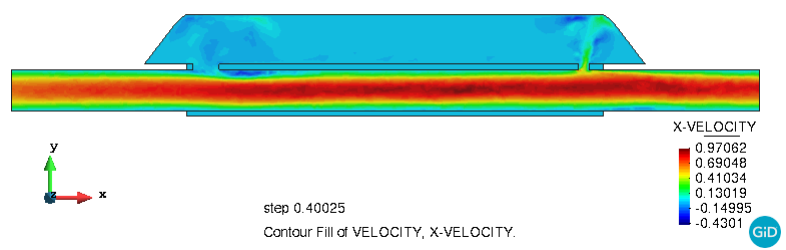

(b) $\mathrm{t}=0.4 \mathrm{~s}$

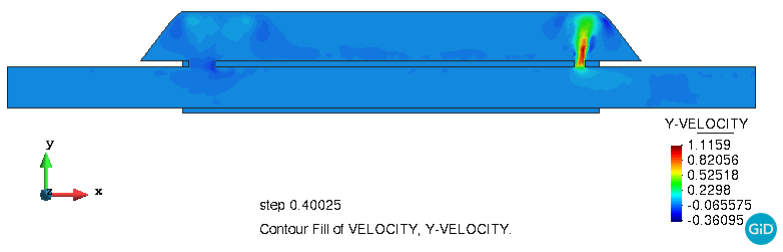

(d) $\mathrm{t}=0.4 \mathrm{~s}$

FIGURE 14 Case 10-4. Velocity fields at different time instances.

Vertical velocity evolution at the tears is shown in Fig. 15 Velocity is measured in the vicinity of the right walls of each tear, so as to capture the behavior of the jet. In comparison with the Case 10-10, a much higher flux through the distal tear is observed here (maximum velocity nearly 3 times higher during the systole and nearly 5 times higher in the secondary peak).

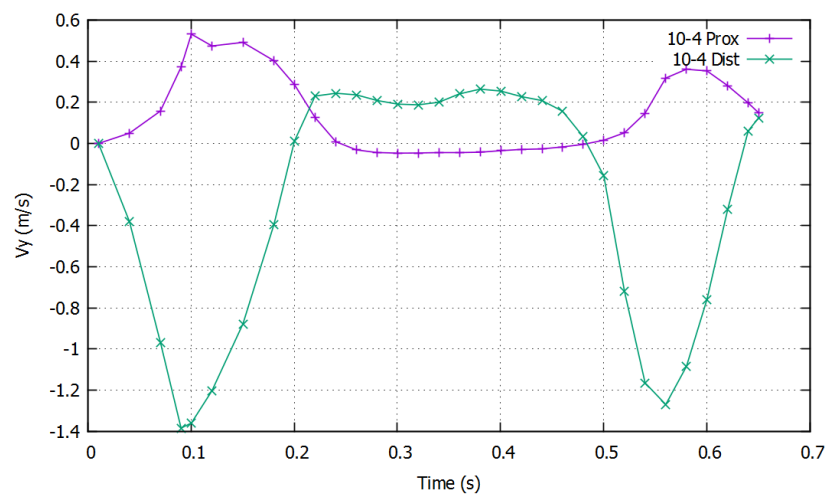

FIGURE 15 Case 10-4. Vertical velocity evolution at proximal and distal tears. 


\section{Flap deformation}

Fig. 16 (a) shows time evolution of the vertical displacement of the flap. One can see that maximum displacement is reached at $\mathrm{t}=0.25 \mathrm{~s}$ and equals approximately $0.06 \mathrm{~mm}$ (some $20 \%$ higher than in the Case 10-10). Fig. 16 (b) shows the distribution of Y-displacement in the flap as the deformed geometry at the end of the simulation $(\mathrm{t}=0.88 \mathrm{~s})$. The displacements were magnified by the factor of 100. One can that the intimal flap undergoes larger expansion in the vicinity of the distal tear. Evolution of the normal stresses in the intimal flap is shown and discussed in Section 4.4

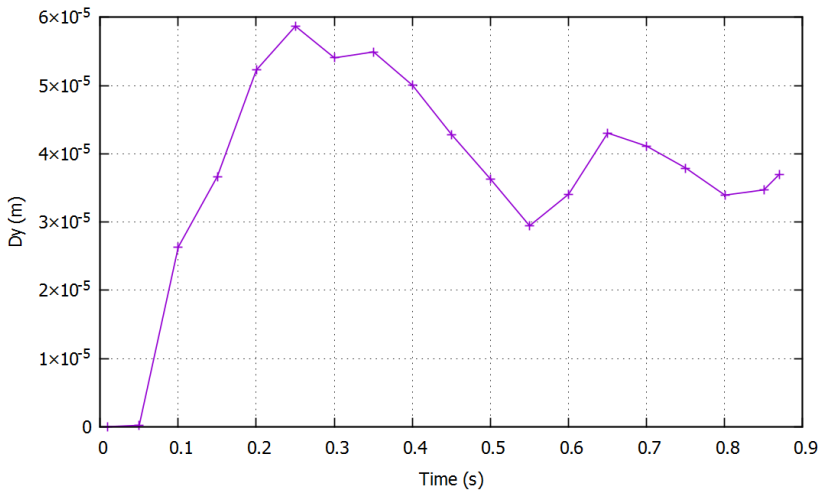

(a) Maximum vertical displacement

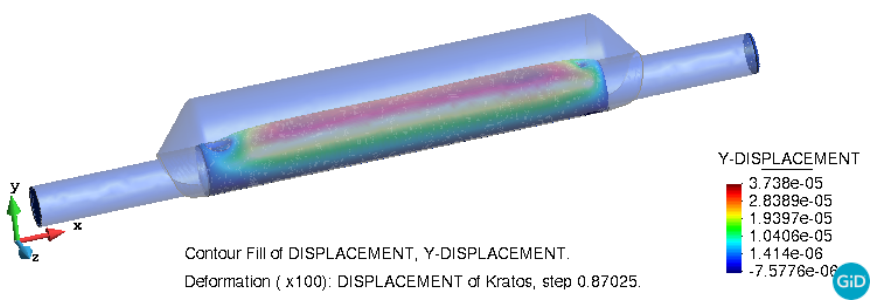

(b) Deformed solid at $\mathrm{t}=0.87 \mathrm{~s}$. Magnif. $\mathrm{x} 100$

FIGURE 16 Case 10-4. Flap deformation.

\section{3 | Case 10-0}

Additionally to the above-described cases characterized by the presence of two tears, we have analyzed a configuration with no distal tears. Proximal tear with a diameter of $10 \mathrm{~mm}$ was considered. Inlet and outlet conditions coincide with those of the previous cases.

\section{Fluid flow}

Fig. 17 shows pressure evolution at the numerical sensors. One can see that slight pressure instability appears at all the locations except for the TL distal sensor. Pressures at the three sensors (TL/FL proximal and FL distal) are nearly identical, with a maximum value of around $16700 \mathrm{~Pa}$, which is larger than the maximum pressures observed in the cases characterized by the presence of a distal tear.

Fig. 18 shows velocity distribution at $\mathrm{t}=0.1$ and $\mathrm{t}=0.4 \mathrm{~s}$. Comparing the observed fields with the formerly analyzed cases, one can see that at both instances, fluid re-circulation is observed at the tear. A small jet entering FL emerges at the right corner of the tear, while a slightly wider jet in the opposite direction enters the $\mathrm{TL}$ at the left corner of the tear during the systole $(\mathrm{t}=0.1)$. At $\mathrm{t}=0.4$ the situation is opposite. The velocity value of the jets is much smaller than in the formerly analyzed cases. Additionally, one can observe vortices appearing in the TL (see Fig. 18 d)). Horizontal velocity pattern still remains nearly unaffected.

\section{Flap deformation}

Fig. 19 (a) shows time evolution of the vertical displacement of the the flexible flap measured at the location where maximum displacement was observed $((\mathrm{x}, \mathrm{y}, \mathrm{z})=(0.32,0.099,0))$. One can see that the maximum displacement and the entire curve is very similar to that of the Case 10-4. Maximum displacement is reached at $\mathrm{t}=0.25 \mathrm{~s}$ and equals approximately $0.06 \mathrm{~mm}$. The vertical displacement at the end of the simulation is around $0.04 \mathrm{~mm}$ (some $10 \%$ larger than in the Case 10-4). Fig. 19 displays the distribution of Y-displacement in the flap as the deformed geometry at the end of the simulation ( $\mathrm{t}=0.88 \mathrm{~s})$. One can see a considerably higher expansion of the intimal flap at the distal part than at the proximal one. The pattern is completely non-symmetrical. Evolution of the stresses in the intimal flap is shown in Section 4.4 


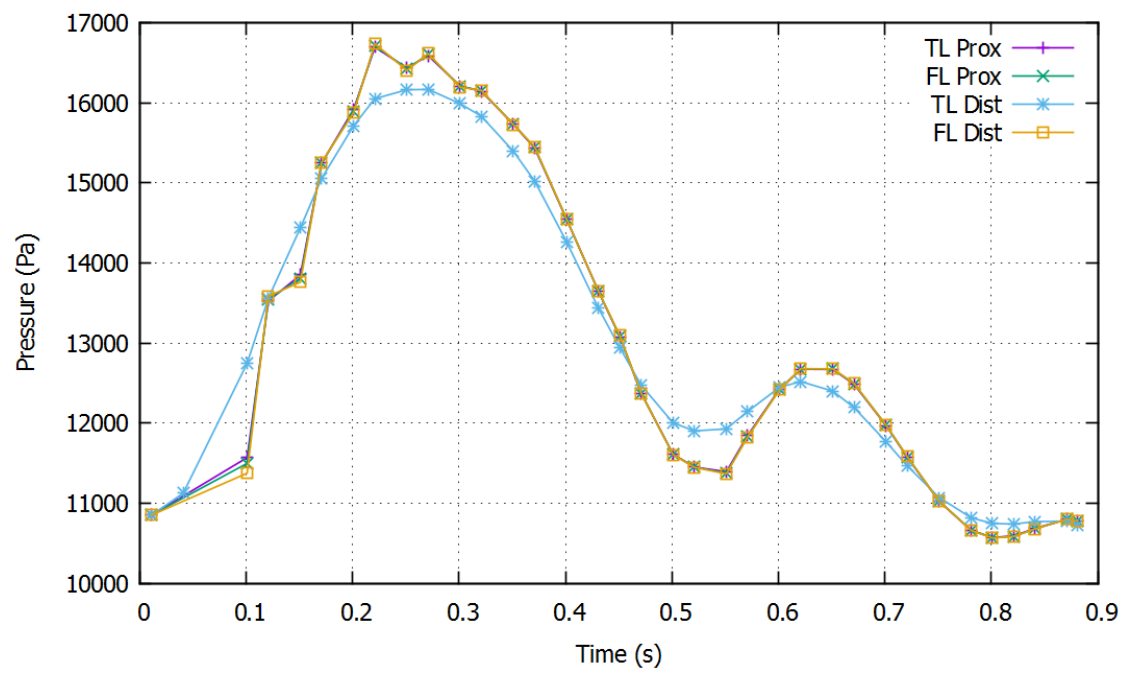

FIGURE 17 Case 10-4. Pressure evolution at the "numerical sensors".

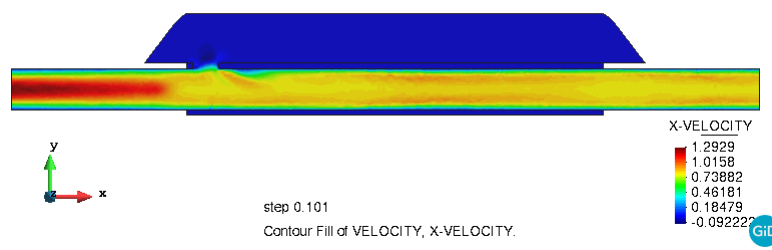

(a) $\mathrm{t}=0.1 \mathrm{~s}$

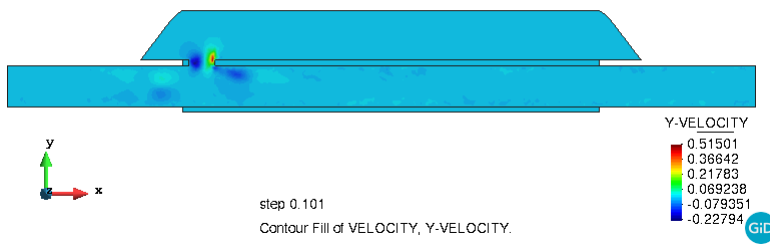

(c) $\mathrm{t}=0.1 \mathrm{~s}$

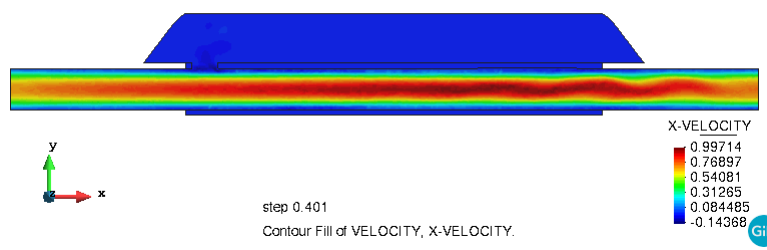

(b) $\mathrm{t}=0.4 \mathrm{~s}$

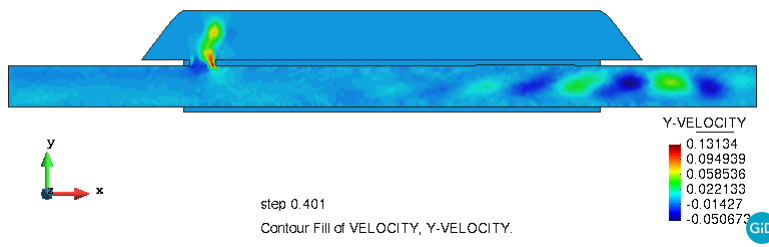

(d) $\mathrm{t}=0.4 \mathrm{~s}$

FIGURE 18 Case 10-0. Velocity fields at different time instances.

\section{4 | Discussion}

\section{Pressure}

In all the analyzed cases pressure measured in 4 locations (in the vicinity of the tears, on TL and FL sides) are similar to the outlet pressure. For small distal tears the difference between TL distal pressure and the pressure at the rest of the locations becomes more pronounced. Large tears ensure nearly uniform pressure distribution between the lumina. In case of the configuration consisting of a single tear, pressure oscillations are observed.

Pressure differences between TL and FL may indicate conditions of aortic growth arising 2 . Aortic fenestration is a method for decompressing the hypertensive false lumen by creating a hole in the distal part of the dissection flap 29 so as to reduce the FL/TL pressure difference. To get the insight of these differences in the cases studied here, pressures were measured in the FL and TL also in the midpoint locations. The midpoint pressures in FL and TL observed in the three analyzed configurations are provided in Table 3 . One can see that the maximum pressure difference between the FL and TL manifests in the case of no distal tear (pressure difference of $390 \mathrm{~Pa}$ ). Introducing a second tear results in diminished pressure difference. Small distal 


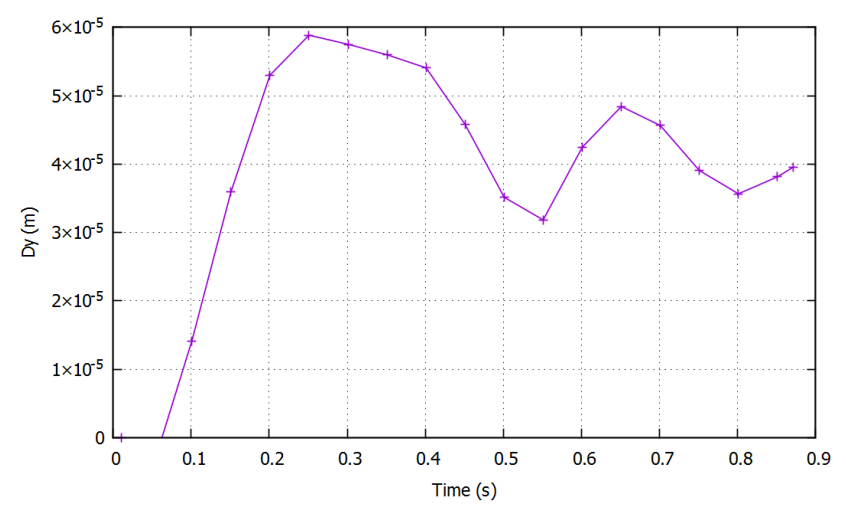

(a) Vertical displacement at Point $\mathrm{M}$

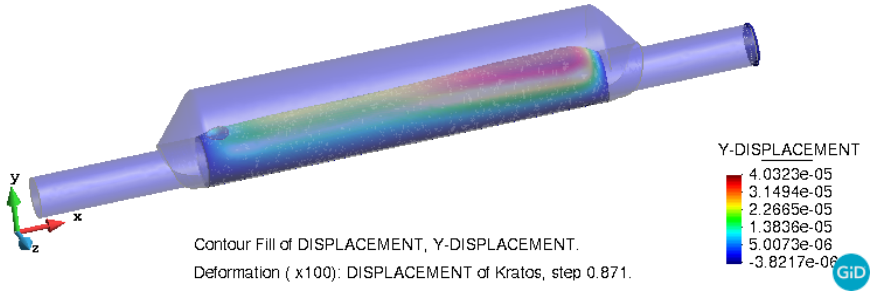

(b) Deformed solid at $\mathrm{t}=0.87 \mathrm{~s}$. Magnif. $\mathrm{x} 100$

FIGURE 19 Case 10-0. Flap deformation.

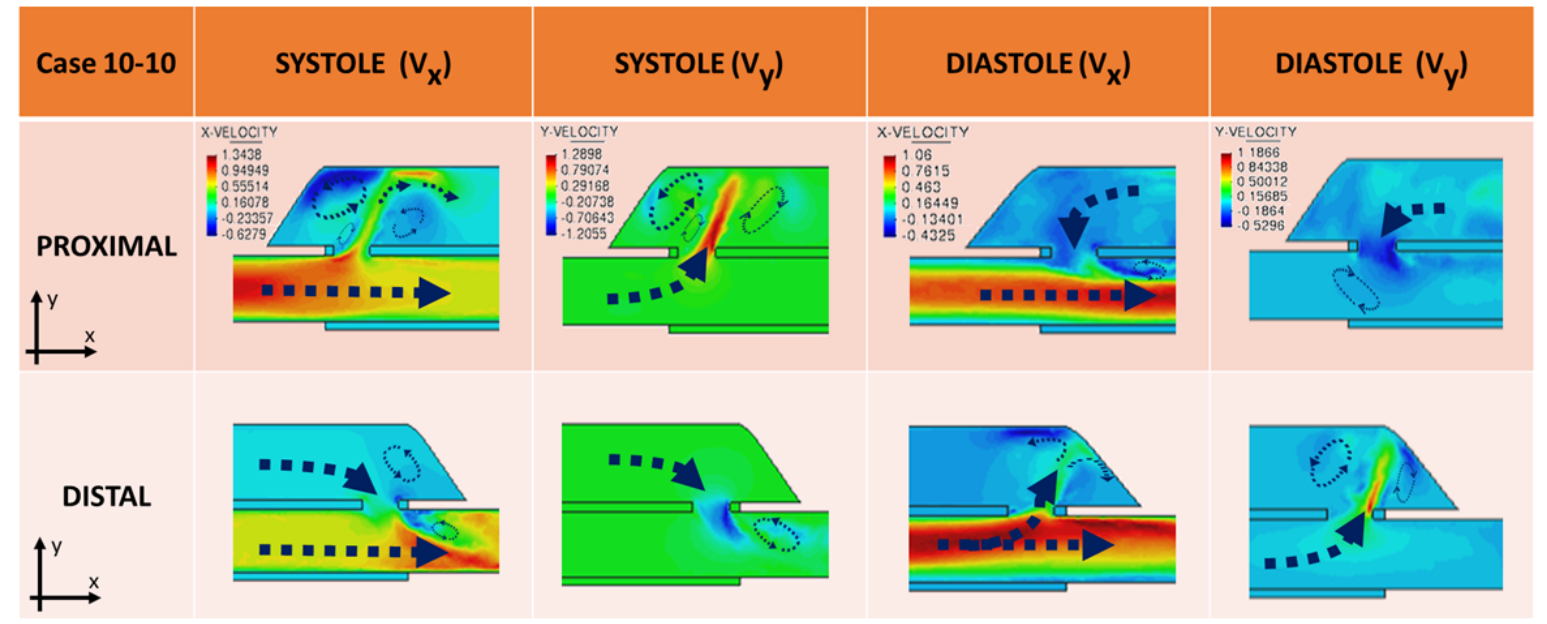

FIGURE 20 Case 10-10. Velocity in the vicinity of the tears and the characteristic features of the flow.

tear reduces the pressure difference to $90 \mathrm{~Pa}$. Large distal tear results in negligible pressure difference between the true and false lumen. This observation confirms that the small tear size may produce insufficient reduction of the FL pressure. This is in line with the conclusion made in ${ }^{30}$, where it was stated that the pressure difference between the TL and the FL is inversely proportional to the cross-sectional area of the exit tear.

\section{Velocity of the flow}

Detailed view of the flow velocity in the vicinity of the tears is shown in Figs. 20 and 22 for Case 10-10, Case 10-4 and case 10-0, respectively. In case of two tears the flow enters the FL via the proximal tear and exits the FL via the distal tear during systole, while during the diastole the flow enters the FL via distal tear and exits the FL via the proximal tear. The jets passing through the tear are typically shifted in the downstream direction from the center of the tear, especially in case of large tears. In case of a single tear, the flow enters and exits through the tear simultaneously creating flow re-circulation. Vortex structures appear in the flow. In all the analyzed cases horizontal velocity in the true lumen remains nearly unaffected by the tear flow. Even though, the velocity of the vortices is relatively low, the vorticity may affect the haemodynamics in the suprarenal and iliac arteries. This phenomenon requires further investigation. 


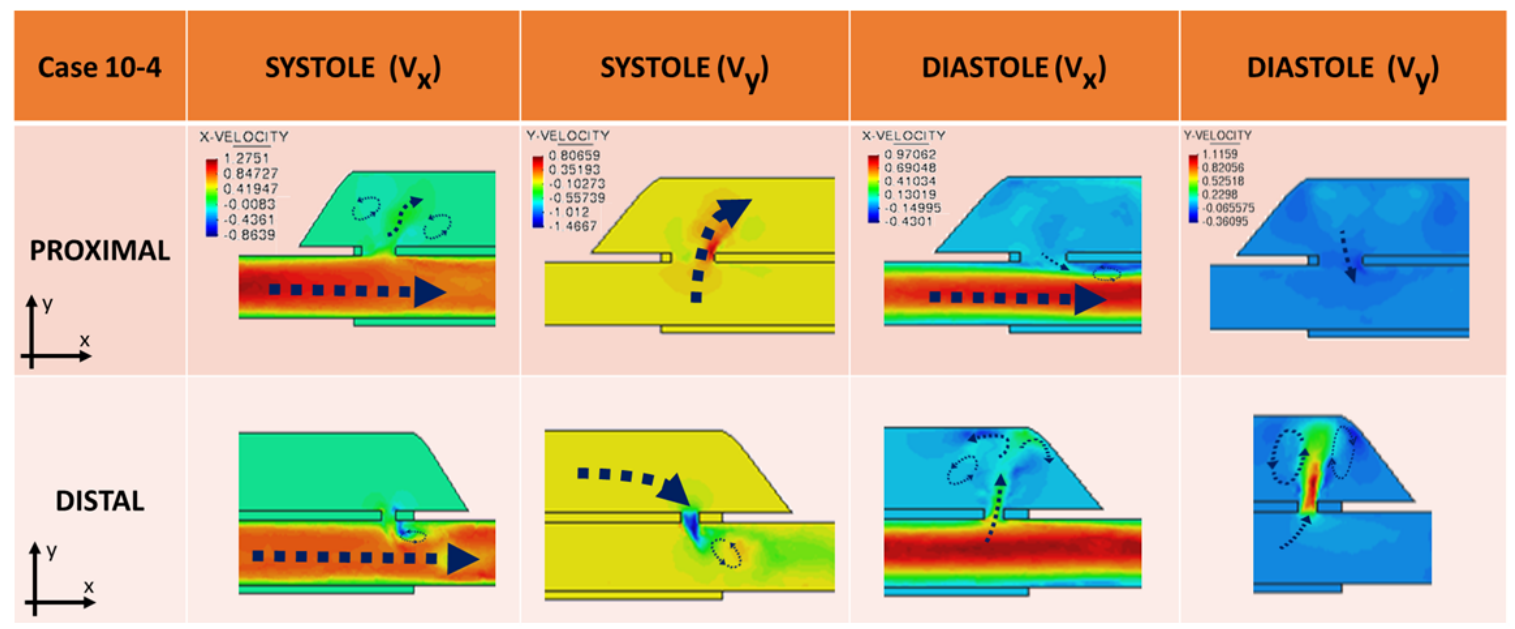

FIGURE 21 Case 10-4. Velocity in the vicinity of the tears and the characteristic features of the flow.

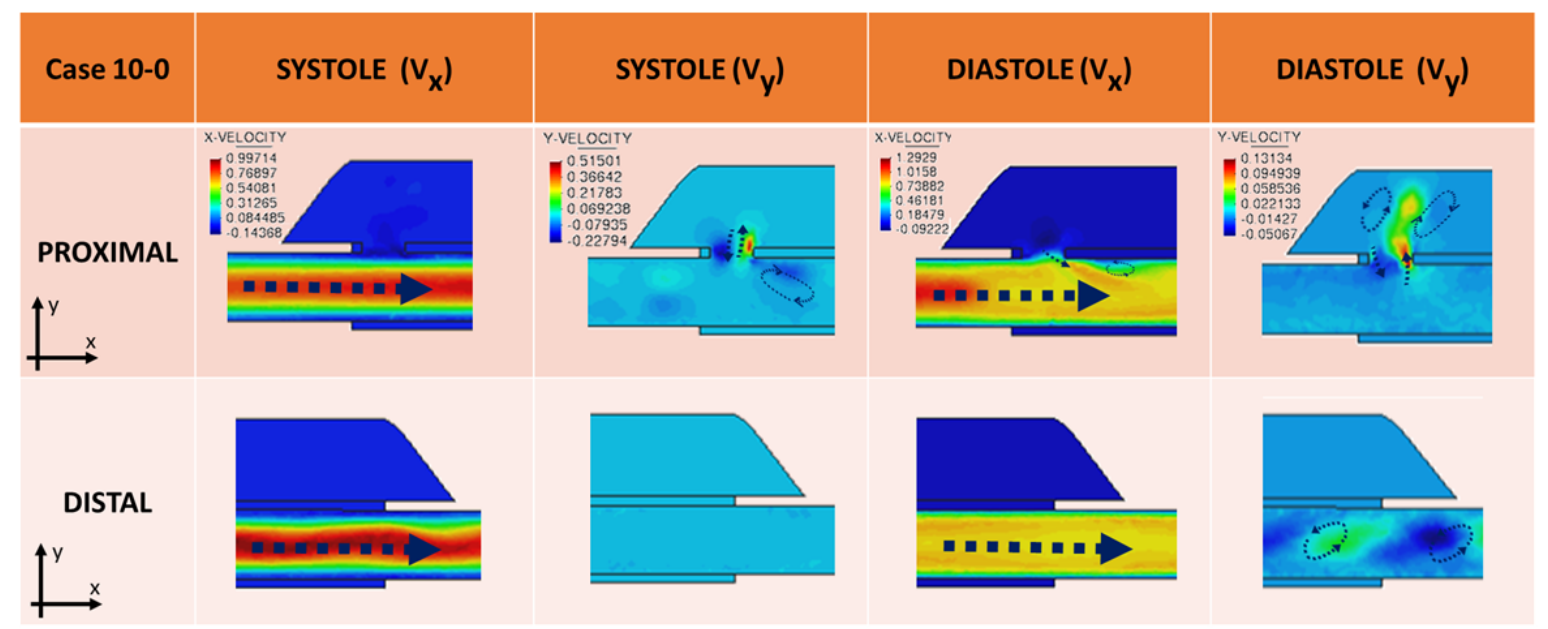

FIGURE 22 Case 10-0. Velocity in the vicinity of the tears and the characteristic features of the flow.

\section{Displacements and stresses}

Evolution of normal stress $\left(\sigma_{x x}\right.$ and $\left.\sigma_{z z}\right)$ for the three analyzed cases are shown in Figs. 23 (a) and 23 (b) One can see that the size of the distal tear has a slight influence on the maximum stress value, however in case of the large tear the difference between the maximum and minimum stresses (amplitude) becomes reduced. In terms of displacements (Fig.23 (c)), small distal tear has nearly no effect (the results with and without the distal tear are nearly identical). However, large distal tear reduces the maximum displacement by $20 \%$. This means that a small tear might provide only a minor reduction in the stresses of the intimal flap, confirming the observation made when analyzing the effect of the tear size upon the pressure difference between the lumina.

Observing the deformation distribution in the flap in case of no distal tear, one can see that the maximum expansion coincides with the area where fenestration is usually carried out.

\section{5 | SUMMARY AND CONCLUSIONS}

In this work a monolithic Arbitrary Lagrangian/Eulerian fluid-structure interaction model developed and implemented by the authors was applied to the simulation of fluid flow in Aortic Dissection. Elastic intimal flap was considered. The model allowed 


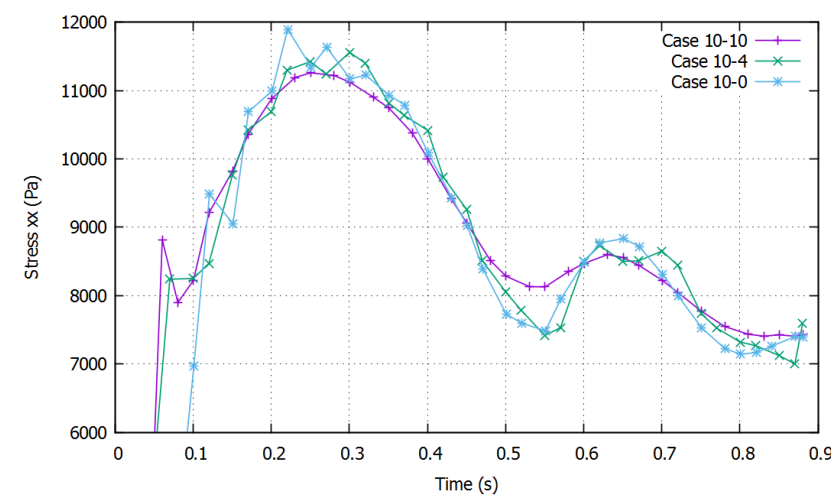

(a) Evolution of $\sigma_{x x}$

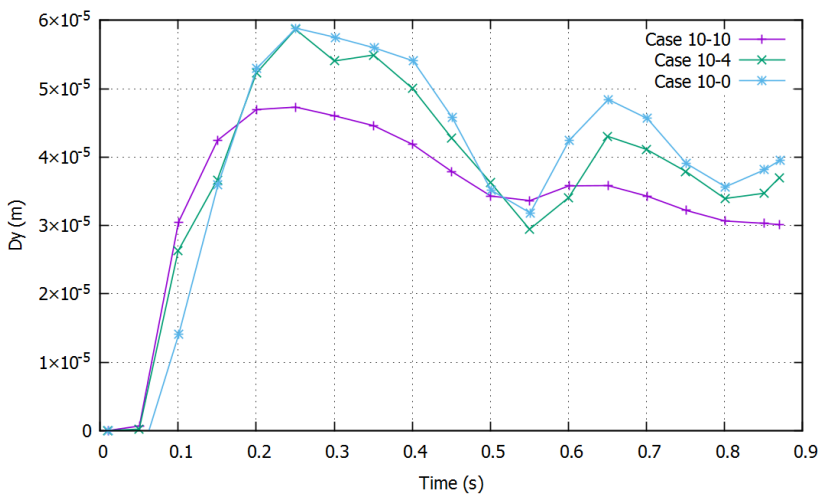

(c) Evolution of vertical displacements

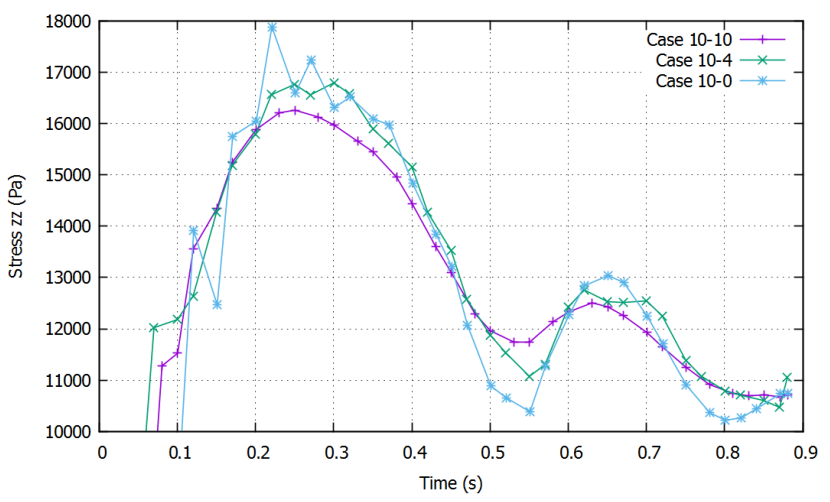

(b) Evolution of $\sigma_{z z}$

FIGURE 23 Comparison of the three cases (Case 10-10, 10-4, 10-0). Stresses and displacements.

\begin{tabular}{l|ccc|ccccc}
\hline Case & $D_{y}(\mathrm{~mm})$ & \multicolumn{2}{c}{ Stress $(\mathrm{Pa})$} & \multicolumn{2}{c}{ Jet $V_{y}$ systole/diastole $(\mathrm{m} / \mathrm{s})$} & \multicolumn{2}{c}{ Midpoint pres. $(\mathrm{Pa})$} & $\Delta p$ TL/FL $(\mathrm{Pa})$ \\
& & $\sigma_{x}$ & $\sigma_{z}$ & Prox. tear & Dist. tear & TL & FL & \\
\hline $10-10$ & 0.045 & 11250 & 16270 & $1.28 /-0.52$ & $-1.2 / 1.18$ & 16099 & 16125 & 26 \\
$10-4$ & 0.06 & 11540 & 16800 & $0.8 /-0.15$ & $-1.47 / 1.11$ & 16303 & 16397 & 94 \\
$10-0$ & 0.06 & 11960 & 17980 & $0.51 / 0.13$ & N/A & 16390 & 16780 & 390 \\
\hline
\end{tabular}

TABLE 3 Summary of the results (maximum values are provided).

predicting not only the flow data (fluid pressure and velocity), but also the deformation and stresses in the flap. Being monolithic, the model does not require coupling iterations and does not suffer the added mass effect (which manifests in instability of the FSI solution when the density of the fluid is close to that of the solid, which is the case for AD system).

For the cases analyzed, the intimal flap displacement was found to be very low $(\propto 0.05 \mathrm{~mm})$. This deformation is negligible considering the size of the flap and the true lumen. Comparison of the results obtained under assumption of rigid flap with the ones of the elastic flap also led to a conclusion that for the studied material (silicon) the elasticity of the flap has nearly no impact upon the fluid flow. Maximum displacements in case of the configuration consisting of two large tears were found to be about $20 \%$ smaller than in case of small distal tear or no distal tear.

Nevertheless, accounting for solid deformations in the simulation allowed us to get the insights of the effect of the tear size upon the stresses in the flap. It was found that the stresses in the intimal flap decrease in case of configurations involving two 
large tears whole presence of a small distal tear had little impact. In case of a large distal tear, not only the peak values of the axial stress decrease by some $10 \%$, but the amplitude of the oscillation between the systolic and diastolic phases becomes considerably suppressed (by $30 \%$ ). The difference between midpoint pressures at FL and TL was reduced by the factor of 10 in the case of having large distal tear.

Detailed analysis of the flow through the tears revealed that the jets at the tears are typically shifted in the direction of the flow (i.e. downstream). This is an important data that must be taken in consideration in the future experimental and numerical studies as it indicates that velocity varies along the tears and the location of the velocity measurement must be chosen so as to capture the jet. Measurements made in the middle of the tear may be incapable of revealing the true velocity of the jet, particularly for the cases of large tears. We also discovered that at both the systolic and diastolic phases the flow direction via proximal and distal tear is always opposite, i.e. if the flow enters the FL via proximal tear, it simultaneously exits the FL via proximal tear and vice versa.

Overall, the proposed model has proven to be a feasible option for the analysis of the problem at hand. In the future, modeling the entire false lumen channel as a flexible solid must be performed. Analysis of stress distributions in the areas that are affected by the jets emerging through the tears may provide valuable data for the clinical practice in terms of prediction of collapsability in the external part of the false lumen. Analysis of the $\mathrm{AD}$ configuration using the biological data rather than that of a phantom set-up defines the next step to be done in our research. As shown in ${ }^{12}$, in such case the impact of the flap deformation upon the fluid flow through the tears may become significant.

\section{ACKNOWLEDGMENTS}

The authors express their gratitude to Dr. Paula A. Rudenick and Dr. Bart H. Bijnens for providing selected data of the in vitro phantom AD experiments as well as to the Spanish Research Network for Cardiac Computational Modelling (V-Heart) for the interest expressed.

\section{Author contributions}

Investigation: PR ES ND. Model implementation: PR. Geometrical modeling: ES. Data processing: ES ND. Validation: PR ES. Visualization: PR ND. Discussion: PR ES ND. Wrote the paper: PR ES ND

\section{Conflict of interest}

The authors declare no potential conflict of interests.

\section{References}

1. Fattori R, Mineo G, Di Eusanio M. Acute type B aortic dissection: current management strategies. Current opinion in cardiology 2011; 26(6): 488-493.

2. Tolenaar J, Keulen vJ, Trimarchi S, et al. Number of Entry Tears Is Associated With Aortic Growth in Type B Dissections. The Annals of Thoracic Surgery 2013; 96(1): 39 - 42. doi: https://doi.org/10.1016/j.athoracsur.2013.03.087

3. Faure E, Canaud L, Cathala P, Serres I, C. MA, Alric P. Human ex-vivo model of Stanford type B aortic dissection. Journal of Vascular Surgery 2014; 60(3): 767 - 775. doi: https://doi.org/10.1016/j.jvs.2013.06.083

4. Qing KX, Chan YC, Lau SF, Yiu WK, Ting ACW, Cheng SWK. Ex-vivo Haemodynamic Models for the Study of Stanford Type B Aortic Dissection in Isolated Porcine Aorta. European Journal of Vascular and Endovascular Surgery 2012; 44(4): 399-405. doi: 10.1016/j.ejvs.2012.06.004

5. Tsai T, Schlicht M, Khanafer K, et al. Tear size and location impacts false lumen pressure in an ex vivo model of chronic type B aortic dissection. Journal of Vascular Surgery 2008; 47(4): 844 - 851. doi: https://doi.org/10.1016/j.jvs.2007.11.059 
6. Rudenick P, Bijnens B, García-Dorado D, Evangelista A. An in vitro phantom study on the influence of tear size and configuration on the hemodynamics of the lumina in chronic type B aortic dissections. Journal of vascular surgery 2013; 57(2): 464-474.

7. Peelukhana S, Wang Y, Berwick Z, et al. Role of Pulse Pressure and Geometry of Primary Entry Tear in Acute Type B Dissection Propagation. Annals of Biomedical Engineering 2017; 45(3): 592-603. doi: 10.1007/s10439-016-1705-4

8. Marconi S, Lanzarone E, De Beaufort H, Conti M, Trimarchi S, Auricchio F. A novel insight into the role of entry tears in type B aortic dissection: pressure measurements in an in vitro model. The International journal of artificial organs 2017; 40(10): 563-574.

9. Soudah E, Rudenick P, Bordone M, et al. Validation of numerical flow simulations against in vitro phantom measurements in different type B aortic dissection scenarios. Computer methods in biomechanics and biomedical engineering 2015; 18(8): 805-815.

10. Ben Ahmed S, Dillon-Murphy D, Figueroa C. Computational study of anatomical risk factors in idealized models of type B aortic dissection. European Journal of Vascular and Endovascular Surgery 2016; 52(6): 736-745.

11. Wan ab Naim W, Ganesan P, Sun Z, Osman K, Lim E. The impact of the number of tears in patient-specific Stanford type B aortic dissecting aneurysm: CFD simulation. Journal of Mechanics in Medicine and Biology 2014; 14(02): 1450017.

12. Alimohammadi M, Sherwood J, Karimpour M, Agu O, Balabani S, Díaz-Zuccarini V. Aortic dissection simulation models for clinical support: fluid-structure interaction vs. rigid wall models. Biomedical engineering online 2015; 14(1): 34.

13. Tse KM, Chiu P, Lee HP, Ho P. Investigation of hemodynamics in the development of dissecting aneurysm within patientspecific dissecting aneurismal aortas using computational fluid dynamics (CFD) simulations. Journal of biomechanics 2011; 44(5): 827-836.

14. Chen H, Peelukhana S, Berwick Z, et al. Fluid-Structure Interaction Simulations of Aortic Dissection with Bench Validation. Journal of Vascular Surgery 2016; 64(6): 1892.

15. Sherrah A, Callaghan F, Puranik R, et al. Multi-Velocity Encoding Four-Dimensional Flow Magnetic Resonance Imaging in the Assessment of Chronic Aortic Dissection. AORTA Journal 2017; 5(3): 80.

16. Liu D, Fan Z, Li Y, et al. Quantitative Study of Abdominal Blood Flow Patterns in Patients with Aortic Dissection by 4-Dimensional Flow MRI. Scientific Reports 2018; 8(1): 9111. doi: 10.1038/s41598-018-27249-9

17. Van Brummelen E. Added mass effects of compressible and incompressible flows in fluid-structure interaction. Journal of Applied mechanics 2009; 76(2): 021206.

18. Causin P, Gerbeau JF, Nobile F. Added-mass effect in the design of partitioned algorithms for fluid-structure problems. Computer methods in applied mechanics and engineering 2005; 194(42-44): 4506-4527.

19. Ryzhakov P, Rossi R, Idelsohn S, Oñate E. A monolithic Lagrangian approach for fluid-structure interaction problems. Computational mechanics 2010; 46(6): 883-899.

20. Idelsohn S, Marti J, Limache A, Oñate E. Unified Lagrangian formulation for elastic solids and incompressible fluids: application to fluid-structure interaction problems via the PFEM. Computer Methods in Applied Mechanics and Engineering 2008; 197(19-20): 1762-1776.

21. Hron J, Turek S. A monolithic FEM/multigrid solver for an ALE formulation of fluid-structure interaction with applications in biomechanics. In: Springer. 2006 (pp. 146-170).

22. Passerini T, Quaini A, Villa U, Veneziani A, Canic S. Validation of an open source framework for the simulation of blood flow in rigid and deformable vessels. International journal for numerical methods in biomedical engineering 2013; 29(11): 1192-1213.

23. Ryzhakov P. A modified fractional step method for fluid-structure interaction problems. Revista Internacional de Métodos Numéricos para Cálculo y Diseño en Ingeniería 2017; 33(1-2): 58-64. 
24. Ryzhakov P, Marti J. A unified Arbitrary/Lagrangian Eulerian Finite Element model for the simulation of fluid-structure interaction in problems involving flows in flexible pipes. In: Proc. VIII International Conference on Computational Methods for Coupled Problems in Science and Engineering.

25. Truesdell C, Noll W. The non-linear field theories of mechanics. In: Springer. 2004 (pp. 1-579).

26. Marti JM, Idelsohn SR, Limache AC, Calvo NA, D’elia J. A Fully Coupled Particle Method For Quasi Incompressible Fluid-Hypoelastic Structure Interactions. 2006.

27. Dadvand P, Rossi R, Oñate E. An object-oriented environment for developing finite element codes for multi-disciplinary applications. Archives of computational methods in engineering 2010; 17(3): 253-297.

28. Rudenick P, Bordone M, Bijnens B, et al. A multi-method approach towards understanding the pathophysiology of aortic dissections-the complementary role of in-silico, in-vitro and in-vivo information. In: Springer. ; 2010: 114-123.

29. Hartnell G, Gates J. Aortic fenestration: a why, when, and how-to guide. Radiographics 2005; 25(1): 175-189.

30. Berguer R, Parodi J, Schlicht M, Khanafer K. Experimental and Clinical Evidence Supporting Septectomy in the Primary Treatment of Acute Type B Thoracic Aortic Dissection. Annals of Vascular Surgery 2015; 29(2): 167 - 173. doi: https://doi.org/10.1016/j.avsg.2014.10.001 